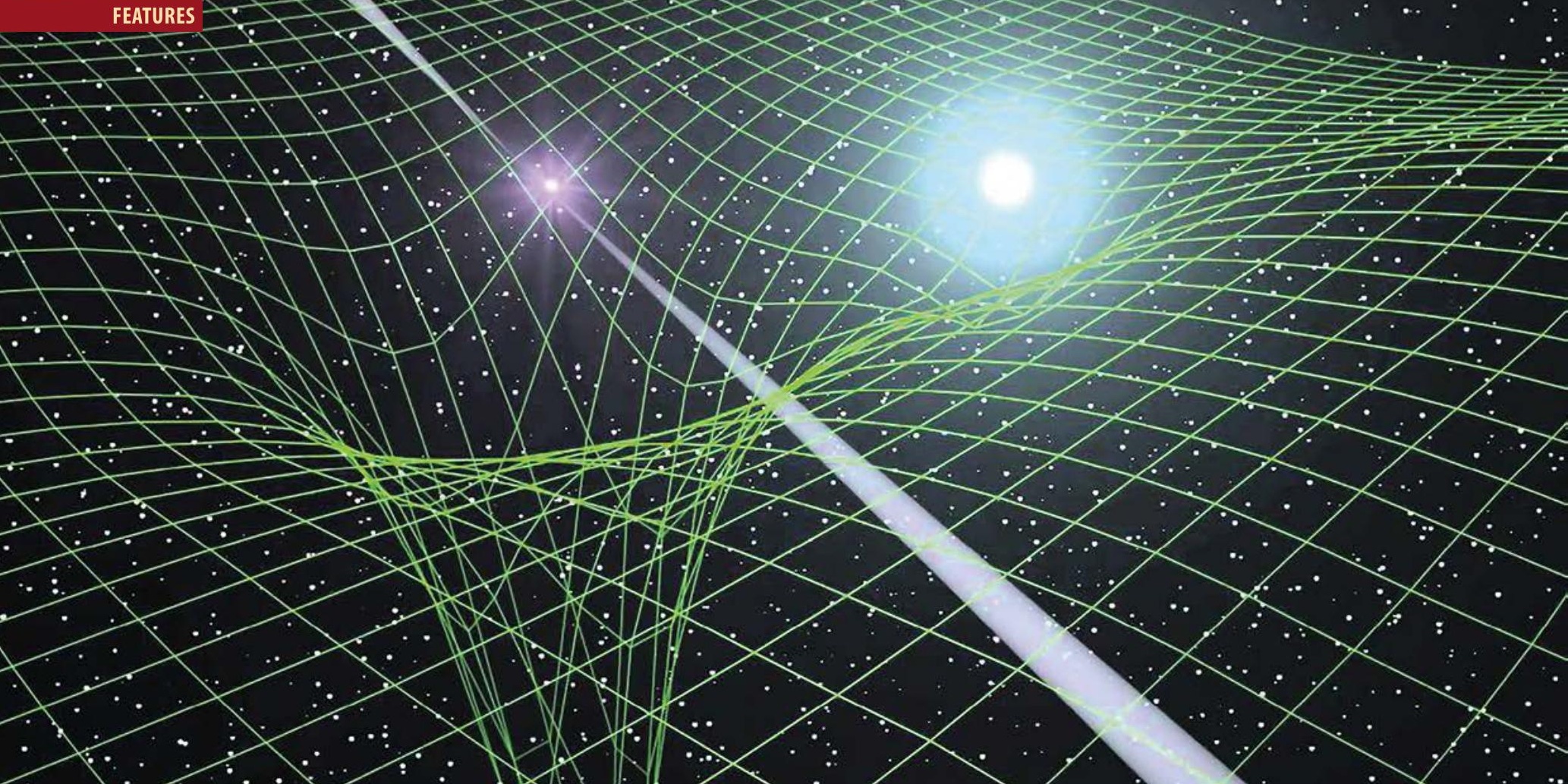

\title{
GRAVITATIONAL PHYSICS: THE BIRTH OF A NEW ERA
}

_ Mairi Sakellariadou - DOI: https://doi.org/10.1051/epn/2017501

We live the golden age of cosmology, while the era of gravitational astronomy has finally begun. Still, fundamental puzzles remain. Standard cosmology is formulated within the framework of Einstein's General theory of Relativity. Notwithstanding, General Relativity is not adequate to explain the earliest stages of cosmic existence, and cannot provide an explanation for the Big Bang itself. Modern early universe cosmology is in need of a rigorous underpinning in Quantum Gravity.

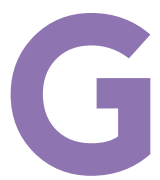

eneral Relativity, the well-known Albert Einstein's theory, describes warps in space-time, and accounts for the large-scale dynamics of the cosmos - the dynamics of galaxies and clusters of galaxies, the dynamics of black holes and even the dynamics of our Universe - with an extraordinary accuracy. General Relativity describes the way massive objects curve space-time, turning a flat landscape into curved scenery of hills and basins. NASA's Gravity Probe B mission [1], launched in April 2004, confirmed two fundamental predictions of the theory of General Relativity. With the help of very precise gyroscopes, Gravity Probe B measured the warping of space-time around the Earth, as well as the amount of space-time the Earth pulls with it as it rotates. These effects are predicted by the theory of General Relativity to be equal to 6606 milliarcsec/year and 39.2 milliarcsec/ year, respectively, while Gravity Probe B measurements lead to $6602 \pm 18 \mathrm{milliarcsec} /$ year and $37.2 \pm 7.2 \mathrm{milliarcsec} / \mathrm{year}$, respectively [2]. The accuracy is indeed remarkable.

In 1998, astronomical observations revealed that the Universe undergoes an accelerated expansion, while naively one would expect the expansion to be slowing down. Within the framework of General Relativity, the late acceleration originates from dark energy, with the simplest option being the cosmological constant, first introduced by Einstein. However, in order to explain the current acceleration of the Universe, the required value of the cosmological constant must be incredibly small. Alternatively, one may consider a large-distance modification to Einstein's theory of General Relativity and relax the hypothesis of dark energy. The explanation of the late-era accelerated expansion and the unknown nature of dark energy remain open questions that challenge our understanding of the Universe and question the validity or completeness of our theoretical models. 
Relativity is not just an abstract mathematical theory with no applications in our daily life. Special and General Relativity play an important role in the Global Positioning System (GPS) which requires nanosecond accuracy. Combining Special and General Relativity effects implies that the atomic clocks on board of each of the GPS satellites tick faster than identical clocks on the ground by about 38 microseconds per day. If these effects were not taken into account then errors in global positions would accumulate at a rate of about 10 kilometers per day, rendering the system worthless for navigation purposes.

The theory of General Relativity predicts the existence of ripples in the fabric of space-time - called gravitational-waves - produced during a violent event of an astrophysical or cosmological origin in the faraway Universe. The existence of gravitational-waves was first demonstrated by Taylor and Weisberg who shown that the orbit of the Hulse-Taylor Pulsar (PSR 1913+16) shrinks slowly over time - only 40 seconds over 30 years - as the result of energy release in the form of gravitational-waves [3]. In 1993, the Nobel Prize in Physics was awarded jointly to Russell A. Hulse and Joseph H. Taylor Jr. "for the discovery of a new type of pulsar, a discovery that has opened up new possibilities for the study of gravitation". The hunt for gravitational-waves lasted several years and the announcement of the first detection in 2016 came 100 years after Einstein's prediction of their existence [4].

According to the theory of General Relativity, a compact binary system loses energy through gravitational-waves emission, bringing the pair of compact objects closer to each other, until a merger takes place leading to the formation of a single more massive spinning compact object and the release of energy in the form of burst of gravitational-waves. On September 14,2015, the two detectors of the Laser Interferometer Gravitational-Wave Observatory (LIGO) [5] - located in Livingston, Louisiana, and Hanford, Washington, USA - simultaneously detected a transient gravitational-wave signal, hence confirming a major prediction of Einstein's 1915 theory of General Relativity. These gravitational-waves were produced during the final fraction of a second of the merger of two black holes of roughly 29 and 36 solar masses - more massive than known stellar-mass black holes - which took place 1.3 billion years ago, resulting in the formation of a single black hole of 62 solar masses and the release of an energy of 3 solar masses times the speed-of-light squared, in the form of gravitational-waves [6].

The era of gravitational astronomy has then begun. Underpinning this breakthrough is a technological triumph of breathtaking capability. LIGO was able to detect movements in objects equivalent to the width of a human hair at the distance to the nearest star. The disturbances are tiny and their detection requires an exquisite sensitivity. The LIGO interferometers found a way to make measurements that go beyond the limits imposed by the Heisenberg principle of quantum physics. On the $3^{\text {rd }}$ October 2017, the Royal Swedish Academy of Sciences has decided to award the Nobel Prize in Physics 2017 with one half to Rainer Weiss and the other half jointly to Barry C. Barish and Kip S. Thorne - all three members of the LIGO/VIRGO
Collaboration - "for decisive contributions to the LIGO detector and the observation of gravitational waves". In announcing the award, the Royal Swedish Academy called it "a discovery that shook the world."

Gravitational-waves carry information - otherwise inaccessible to us - from a variety of sources (e.g., phase transitions in the early Universe, topological defects as relics of a previous more symmetric stage of the Universe, an early inflationary era which is a paradigm proposed to cure some of the pathologies that plague the standard hot Big Bang cosmological model, or mergers of compact binary systems formed by neutron stars or black holes), opening a new window on our cosmic origins, hence widening our understanding of astrophysics, cosmology, as well as fundamental physics. Gravitational-waves can be considered as the most promising cosmic relic to probe the unknown aspects of the early Universe, as they can provide information during the period that the Universe was opaque to electromagnetic radiation.

Gravitational physics is now entering a golden age of discovery. The Advanced Virgo detector [7] - located in Cascina, Italy - joined the Advanced LIGO twin interferometers on August 1,2017. Other detectors are planned to join the network: KAGRA is being built in Japan [8], and LIGO India has been recently approved. The Microscope satellite [9], aimed to test the equivalence between gravitational and inertial mass, has been successfully launched and is now taking data. Following the highly successful LISA Pathfinder mission, the European Space Agency has selected the space-based laser interferometer, LISA [10], for its third large class mission, due for launch in 2034. LISA will open a gravitational window on the Universe in the low-frequency regime, providing the closest view of the early stages of the Universe, from its smallest scales near the horizons of black holes, all the way to cosmological scales. There are also plans for the third-generation gravitational-wave, such as the Einstein Telescope [11] or the Cosmic Explorer, reaching unprecedented sensitivities, overcoming current limitations imposed by thermal noise and quantum-optical noise. These detectors will be roughly a factor of 10 more sensitive in strain than the current generation, and be able to detect binary black hole mergers beyond a red-shift of 20 .

Despite its enormous success, the theory of General Relativity has its limitations. The theory describes very well the dynamics of the Universe through Einstein's field equations. Running these dynamic equations backwards in time, there is a finite time in the past when the Universe must have been in a state of ultrahigh curvature and mass density. This is the Big Bang era, which took place about 13.8 billion years ago and is considered as the beginning of our observable Universe. At that early time, all matter and energy of our present Universe were confined into a tiny sub-atomic region of space, and the classical theory of General Relativity cannot provide the mathematical tools and physical concepts that will allow us to describe the behaviour of gravity under such extreme curvature and density conditions. Indeed, once we zoom into the microcosm, the behaviour and position of sub-atomic particles become uncertain as we enter 
the realm of Quantum Mechanics, characterised by uncertainties and probabilities. To describe the Big Bang era, a quantum theory of gravity is required; such a theory is expected to cure the singularity which plagues the classical theory.

To address questions, such as what is meant by the origin of the Universe, what was the mechanism that led to its creation, whether its birth was an accidental process and whether it could have been avoided, or even what was before the Big Bang phase, requires an understanding about the state, structure and dynamics of space-time itself. Quantum Gravity is the theory aiming at explaining the shape of space-time and understanding its invisible structure, which we perceive as the force of gravity and which determines our own trajectories. Hence, modern early Universe cosmology is in the need of a rigorous underpinning in Quantum Gravity, the long-sought theory that will offer the appropriate framework to address fundamental questions such as the origin of the space-time continuum and its effective dynamics, and the pictures of the earliest moments of the Universe which emerge.

Different Quantum Gravity proposals have been made and progress has been constantly achieved. These proposals belong to two classes; they are either top-down (guess the structure of space-time at the Planck scale and deduce its consequences at low-energy scales) or bottom-up (deduce the structure of spacetime at the Planck scale from our knowledge at low-energy scales at which colliders operate). In the former class belong approaches like String/M-theory or a variant of non-perturbative approach to Quantum Gravity, like Loop Quantum Gravity [12], Group Field Theory [13], Causal Sets [14], or Causal Dynamical Triangulations [15]. In the latter one belong the proposal of Non-Commutative Spectral Geometry [16] and that of Asymptotic Safety [17]. At present, Quantum Gravity has spawned many new ideas aimed at augmenting or replacing General Relativity as a theory of gravity. The low-energy limit of such Quantum Gravity theory will be the classical theory of General Relativity.

Applied to cosmology, these ideas involve dramatic departures from the standard cosmological model [18], such as bouncing cosmological models, where the Universe undergoes a long, pre-big bang contracting phase which then bounces into the expanding Universe we observe today. Another possibility is an early era of accelerated expansion without the need for a special mechanism such as inflation. These ambitious attempts to model the very early Universe mathematically have been accompanied by plans for observational tests. Searching for physical remnants of very early Universe, not to mention a possible pre-big bang phase, are enormously challenging, but gravitational-waves are acknowledged as the most promising. At present, even though we are not yet in a position to make any definite statement as to which - if any -Quantum Gravity proposal is viable, it is fair to say that a great progress has been made towards the goal to unify gravity with sub-atomic forces. Early Universe cosmology can shed some light on the correct Quantum Gravity theory, unweaving the fabric of the Universe and revealing the very early stages of its infancy [19].

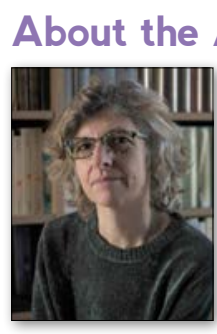

\section{uthor}

Mairi Sakellariadou, Professor of Theoretical Physics, King's College London. Research in Early Universe Theoretical Cosmology, Quantum Gravity/Cosmology (String/Brane Cosmology, Loop Quantum Cosmology, Group Field Cosmology, Non-Commutative Geometry), Gravitational-Waves, Modified Gravity, Topological Defects.

\section{MEMBER OF THE:}

- LIGO Scientific Collaboration, GEO600 Experimen, MoEDAL at LHC Collaboration, LISA Cosmology working group, Einstein Space Team, EUCLID Consortium, Virgo Ego Scientific Forum - Chair of the Gravitational Physics Division of the European Physical Society

- President of the Hellenic Society Relativity, Gravitation and Cosmology

- Advisory Editor for Springer Verlag books

- Co-editor of Europhysics Letters

- Member of the Editorial Advisory Board of Classical and Quantum Gravity

- Emily Noether Visiting Fellow at the Perimeter Institute, Canada - Member of the Advisory Committee of the Research and Training Group “Models of Gravity”, Germany

\section{References}

[1] https://www.nasa.gov/mission_pages/gpb/

[2] C. W. F. Everitt, D. B. DeBra, B. W. Parkinson, J. P. Turneaure, J. W. Conklin, M. I. Heifetz, G. M. Keiser and A. S. Silbergleit et al., Physical Review Letters 106 (2011) 221101

[3] J. H. Taylor and J. M. Weisberg, Astrophysical Journal 253 (1982) 253

[4] A. Einstein, Sitzungsber. Preuss. Akad. Wiss. Berlin (Math.Phys.) (1916) 688

[5] http://www.ligo.org/

[6] B. P. Abbott et al. (LIGO Scientific Collaboration and Virgo Collaboration), Physical Review Letters 116 (2016) 061102.

[7] http://www.virgo-gw.eu/

[8] http://gwcenter.icrr.u-tokyo.ac.jp/en/

[9] https://microscope.cnes.fr/en/MICROSCOPE/index.htm

[10] http://sci.esa.int/lisa/

[11] http://www.et-gw.eu/

[12] A. Ashtekar, New J.Phys. 7 (2005) 198

[13] D. Oriti, in Mathematical and Physical Aspects of Quantum Gravity, B. Fauser, J. Tolksdorf and E. Zeidler, eds., Birkhaeuser, Basel (2006).

[14] L. Bombelli, J. Lee, D. Meyer, and Rafael D. Sorkin, Physical Review Letters 59 (1987) 521.

[15] J. Jurkiewicz, R. Loll and J. Ambjorn, Scientific American (July 2008)

[16] A. Connes, J. Mathematical Physics 41 (2000) 3832

[17] R. Percacci, in Approaches to Quantum Gravity: Towards a New Understanding of Space, Time and Matter, D. Oriti, ed. Cambridge University Press (2008)

[18] M. Sakellariadou, Journal of Physics: Conf. Series 880 (2017) 012003

[19] M. Sakellariadou, in Mathematical Structures of the Universe, edited by the Copernicus Center for Interdisciplinary Studies (2014). 